\section{Prevalência de transtornos mentais comuns em professores da rede municipal de Belo Horizonte, Minas Gerais, Brasil}

\author{
Prevalence of common mental disorders \\ among schoolteachers in Belo Horizonte, \\ Minas Gerais, Brazil
}

Sandra Maria Gasparini 1,2

Sandhi Maria Barreto 2

Ada Ávila Assunção 2

\section{Introdução}

${ }^{1}$ Faculdade de Ciências Médicas de Minas Gerais, Belo Horizonte, Brasil. 2 Faculdade de Medicina, Universidade Federal de Minas Gerais, Belo Horizonte, Brasil.

Correspondência A. A. Assunção

Programa de Pós-graduação em Saúde Pública, Faculdade de Medicina, Universidade Federal de Minas Gerais. Av. Alfredo Balena 190, Belo Horizonte, $M G$ 30130-100, Brasil. adavila@medicina.ufmg.br

\begin{abstract}
Research worldwide has shown that schoolteachers are at increased risk of physical and mental stress due to material or psychological difficulties associated with their work. This study aimed to estimate the prevalence of mental disorders among schoolteachers in the municipal school system in Belo Horizonte, Minas Gerais State, Brazil, and to investigate associated work-related factors. A cross-sectional study was carried out including all $1^{\text {st }}$ and $2^{\text {nd }}$ grade schoolteachers in the Northeast region of the city. A self-applied questionnaire with six sets of questions was applied. Common mental disorders in the target population were assessed using the General Health Questionnaire (GHQ-12). 751 of the 792 schoolteachers (94.8\%) participated in the study. Mental disorders were significantly associated with: positive history of violence, deficient workplace conditions and comfort, low creativity and autonomy for development of work, and insufficient time for preparing course work. The results show that mental disorders are an important health problem among schoolteachers and provide some inputs on how working conditions could be improved to relieve teachers' mental strain.
\end{abstract}

Mental Health; Mental Disorders; Faculty; Violence; Schools
O interesse pelo estudo da relação entre saúde mental e trabalho tem crescido nos últimos anos, devido ao aumento da prevalência dos transtornos mentais e dos distúrbios do comportamento em trabalhadores, observado em diversos países ${ }^{1}$. Segundo Jacques 1 , as teorias sobre estresse, as teorias da psicopatologia e da psicodinâmica do trabalho, as teorias com base no modelo epidemiológico e/ou diagnóstico e os estudos sobre subjetividade e trabalho contribuem para identificar fatores ocupacionais implicados nessa relação 2,3,4

Segundo Jacques 1, cerca de 30 a $40 \%$ dos trabalhadores ocupados no mundo apresentam algum tipo de transtorno mental. No Brasil, segundo as estatísticas do Instituto Nacional do Seguro Social (INSS), de 2001, os distúrbios psíquicos ocupam o terceiro lugar entre as causas de concessão de benefício previdenciário, com afastamento do trabalho em tempo superior a 15 dias e de auxílio doença por invalidez.

As transformações técnicas e organizacionais do trabalho vêm-se acelerando e gerando significativas conseqüências para a vida e saúde dos trabalhadores em geral, o que condiciona mudanças no seu perfil de morbi-mortalidade, com aumento de doenças mentais, psicossomáticas, cardiovasculares e osteoarticulares, entre outras. Os fatores psicossociais, definidos pela Organização Internacional do Tra- 
balho (OIT) como aqueles que se referem à interação entre o meio ambiente laboral, o conteúdo do trabalho, as condições organizacionais, e as necessidades e habilidades dos trabalhadores podem influenciar a saúde, o desempenho e a satisfação no trabalho. Entre esses fatores pode-se destacar: a estabilidade no emprego, o salário, as relações sociais no trabalho, a carga e o conteúdo do trabalho com seus desafios, o ambiente físico, a autonomia, a oportunidade de desenvolvimento profissional, o reconhecimento e a valorização profissional 5,6.

De acordo com Abrahão \& Pinho 7, as transformações que se processam no mundo do trabalho, fenômeno que recebe diversas denominações: mundialização, globalização, terceira revolução industrial e tecnológica, evidenciam um novo paradigma de organização das relações econômicas, sociais e políticas. O mundo do trabalho encontra-se sob um processo de reestruturação produtiva e organizacional que altera as novas formas de organização, gestão e controle do trabalho, mudando a sua natureza e estabelecendo novos cenários produtivos. Esse processo de transformação solicita mudanças no perfil dos trabalhadores, apontando para a valorização da polivalência, do comportamento organizacional, da qualificação técnica, da participação criadora, da mobilização da subjetividade e da capacidade de diagnosticar e de decidir. Para atender às novas exigências e desenvolver esse novo perfil, os trabalhadores precisam adquirir novas competências e capacidade para transitar para um novo modo de ser, fazer e pensar.

No setor da educação, o processo de reestruturação produtiva apresenta novas demandas que impulsionam transformações na organização do trabalho docente ${ }^{8}$. A inserção dos indivíduos no mercado de trabalho exige níveis de escolarização cada vez mais elevados. Para atender às novas demandas de organização dos sistemas de produção, espera-se da escola e, principalmente, do docente, a formação de um profissional flexível e polivalente. Entretanto, os engendramentos realizados na tentativa de alcançar maior autonomia no trabalho docente são, muitas vezes, acompanhados por restrições das políticas educacionais com efeitos diretos nas atividades dos professores, no modo de execução de sua atividade e em sua própria saúde 8 .

Na América Latina, as reformas educacionais implantadas a partir de 1990 possuem um duplo enfoque: a educação dirigida à formação da força de trabalho apta às demandas do setor produtivo (gestão do trabalho) e a educação que atenda às demandas da maioria (gestão da po- breza), determinando uma reestruturação da atividade docente com maior responsabilização dos professores e maior envolvimento da comunidade 9 .

Estudos realizados em outros países indicam que há uma relação direta entre o aumento de fatores estressantes no trabalho e níveis elevados de fadiga, alterações do sono, problemas depressivos e consumo de medicamentos 10,11. No Brasil, as transformações na organização do trabalho docente como novas exigências e as competências requeridas modificam a atividade de ensinar, e, por não proverem os meios compatíveis, criam uma sobrecarga de trabalho. Citam-se o volume de trabalho, e a precariedade das condições existentes, mas também à diversidade e à complexidade das questões presentes na sala de aula e, ainda, uma expectativa social de excelência. Os fatores mencionados podem estar na origem de queixas e adoecimento mental na categoria 4,12,13,14,15,16,17,18,19,20,21,22.

Além das mudanças no trabalho docente, as escolas deixaram de representar um local seguro e protegido e incorporaram a violência do cotidiano e do entorno dos estabelecimentos de ensino. A OIT e a Organização das Nações Unidas para a Educação, a Ciência e a Cultura (UNESCO) relatam um aumento progressivo da violência nas instituições escolares em todo o mundo e interpretam a violência escolar como expressão de fenômenos como a globalização e a exclusão social 11,23. A violência no Brasil tornou-se uma situação social grave e esse contexto tem repercussões importantes no trabalho, sendo uma fonte importante de estresse nas escolas.

Neste estudo, propõe-se estimar a prevalência de transtornos mentais em professores da rede municipal de ensino de Belo Horizonte e investigar sua associação com as características do trabalho docente.

\section{Métodos e procedimentos}

\section{Delineamento da pesquisa}

Realizou-se um estudo epidemiológico de corte transversal sobre as condições de trabalho, ambientais e organizacionais, e indicadores selecionados de saúde dos professores do ensino fundamental da rede municipal de Belo Horizonte, desenvolvido em duas etapas: etapa exploratória e etapa de desenvolvimento. O presente estudo descreve a realização do inquérito realizado na Regional Nordeste. 


\section{- Período, local e população do estudo}

A rede municipal de ensino de Belo Horizonte é formada por 182 escolas, divididas em nove regionais. Participaram do estudo todos os professores do 1 o ao 3 o ciclos, que trabalham nas 26 escolas (de um conjunto de trinta) do ensino fundamental da Regional Nordeste nos turnos da manhã e da tarde, do ensino fundamental, totalizando 751 professores. As quatro escolas restantes não foram incluídas devido à sua missão destinada à educação infantil e às especificidades que lhe são decorrentes. Foram excluídos os professores de educação física e os licenciados por qualquer motivo.

\section{- Etapas do trabalho de campo}

a) Etapa exploratória: várias reuniões foram realizadas com a diretoria do Sindicato Único dos Trabalhadores do Ensino (SIND-UTE). Os profissionais da Gerência de Saúde e Perícia Médica (GSPM) foram entrevistados procurando entender o funcionamento do serviço e os problemas enfrentados no diagnóstico e encaminhamento dos casos de licenças médicas dos professores doentes.

b) Etapa de desenvolvimento: o diretor de cada escola recebeu a carta-convite e o termo de consentimento livre e esclarecido ao professor, a carta-convite e o termo de consentimento livre e esclarecido ao diretor da escola e o instrumento da pesquisa para esclarecimento. A adesão dos diretores foi plena. Paralelamente, o SIND-UTE divulgou na categoria os objetivos da pesquisa, facilitando, assim, o posterior contato com o universo pesquisado.

\section{Instrumento de coleta de dados}

Os dados da literatura e os resultados das entrevistas realizadas junto ao SIND-UTE e à GSPM, na etapa exploratória, serviram para a elaboração do questionário que foi aplicado, sem a identificação do sujeito, visando a coletar informações sobre alguns problemas de saúde e trabalho vivenciados pelos professores. O questionário foi construído com seis blocos de questões. O primeiro bloco continha informações sobre as características sócio-demográficas, econômicas, local e tipo de trabalho realizado e hábitos de vida. O segundo referia-se à história profissional no trabalho docente, violência no ambiente de trabalho, relacionamento com colegas, alunos, familiares de alunos e superiores, acesso à escola e modo de deslocamento do professor até a escola, organização, processo e ritmo de trabalho, fiscalização da direção, satisfação, planejamento e tempo para o preparo das atividades. O terceiro e quarto blocos avaliaram a percepção dos professores quanto ao material pedagógico disponível e às condições das salas de aula (iluminação, ventilação e o nível de ruído). O quinto bloco foi composto por 19 perguntas com vistas a identificar a presença de distúrbios da voz, cujos resultados não integram as análises que serão apresentadas adiante, porém serão analisados posteriormente. No sexto bloco, utilizou-se o General Health Questionnaire-12 (GHQ-12), questionário validado, composto por 12 perguntas, visando a identificar a existência de transtornos mentais entre professores.

O General Health Questionnaire (GHQ) foi desenvolvido por David Goldberg em 1972 e concebido como método de rastreamento de transtornos psíquicos no âmbito clínico da saúde geral. Em sua forma original constava de 93 itens. A partir de então, têm sido geradas versões mais curtas (60, 30, 28, 20 e 12 questões). Nos últimos anos, o GHQ tem-se confirmado como um dos melhores instrumentos dentro das técnicas de rastreamento para estudo de base populacional. É um instrumento auto-aplicável, curto, bem aceito pelos respondentes e fácil para responder. O GHQ, na versão utilizada neste estudo, não pretende detectar doenças psiquiátricas graves como a esquizofrenia e a depressão psicótica nem se presta a diagnosticar quaisquer doenças mentais. Busca apenas apontar os problemas na função mental que se apresentam como uma disfunção, deixando a pesquisa diagnóstica para uma abordagem psiquiátrica posterior 24 .

A efetividade de instrumentos como o GHQ tende a variar com a amostragem, o recrutamento, com o modo de questionamento e com as características culturais da população a ser estudada. As diferentes modalidades do GHQ têm sido traduzidas pelo menos em 36 idiomas e utilizadas em mais de cinqüenta estudos de validação, inclusive no Brasil (sensibilidade de $82 \%$ e especificidade de $71,8 \%$ ) para um ponto de corte de 3/4 no GHQ-12 25. Isto é, o indivíduo que apresentar escore igual ou maior que quatro será considerado positivo, alterado em relação à sua função psíquica habitual, com sintoma de transtorno psíquico 25,26.

A fim de garantir níveis mais elevados de padronização na coleta de dados, foram realizadas várias reuniões com o objetivo de orientar e esclarecer dúvidas quanto aos procedimentos do estudo e padronizar as condutas da equipe de campo. 


\section{Procedimentos de coleta de dados}

A coleta de dados foi realizada nas escolas da Regional Nordeste, durante o horário de recreio dos professores ou em horário das reuniões pedagógicas, no período de novembro a dezembro em dias não consecutivos. Para a aplicação dos questionários, os professores foram informados sobre os objetivos da pesquisa, sobre a instituição responsável e sobre o caráter voluntário e sigiloso da participação de cada um. Procedia-se, então, a entrega da carta-convite e Termo de Consentimento Livre e Esclarecido, e do questionário.

O questionário foi entregue em envelope individual e, em função das questões abordadas, para evitar constrangimento aos professores ou para evitar resistência e obter maior adesão ao estudo, adotou-se um formulário, sem identificação do respondente.

Em algumas escolas não foi possível a realização da pesquisa no momento das reuniões pedagógicas. Nesses casos os questionários foram deixados na escola e, posteriormente recolhidos pela equipe de pesquisa. Em seis casos o questionário foi aplicado na residência do professor.

\section{Análise dos dados}

Os dados foram analisados utilizando-se o programa Epi Info 3.3 (Centers for Disease Control and Prevention, Atlanta, Estados Unidos). Inicialmente foi determinada a prevalência de transtorno mental, utilizando-se o ponto de corte 3/4 da escala de Goldberg.

Os professores com e sem transtornos mentais foram comparados em relação ao conjunto de fatores incluídos em cada bloco do questionário: (1) características sócio-demográficas; (2) comportamento relacionado à saúde e morbidade crônica relacionada; (3) inserção no trabalho; (4) carga de trabalho; (5) experiência com a violência na escola; (6) percepção sobre o trabalho; (7) recursos disponíveis para o trabalho escolar; (8) ambiente físico na escola. A magnitude da associação de cada fator com a presença de transtorno mental foi aferida pelo odds ratio e a significância estatística pelo intervalo de confiança de 95\%, obtidos através de regressão logística múltipla.

Para cada bloco de fatores foi realizada, posteriormente, uma análise multivariada a fim de se identificar os fatores associados de forma independente com a presença de transtornos mentais, tendo sido incluídos, em cada modelo final, todos os fatores associados no nível de $p<0,20$. Foram considerados estatisticamente significantes todos os fatores associados no nível de $\mathrm{p}<0,05$. Tendo em vista que cada bloco representa um conjunto de fatores relacionados entre si, mas não necessariamente relacionados a outros blocos, optamos por não fazer o modelo final com as variáveis que permaneceram associadas em cada um dos blocos.

As desagregações de cada variável em cada um dos blocos foram feitas considerando tanto os aspectos relativos à discriminação de categorias distintas como a possibilidade de redução do número ao mínimo possível, para evitar perda de poder estatístico.

O projeto desta pesquisa foi aprovado pelo Comitê de Ética em Pesquisa (COEP) da Universidade Federal de Minas Gerais (UFMG) em 5 de outubro de 2004 (Parecer n. 240).

\section{Resultados}

Dos 813 professores do ensino fundamental diurno do 1o ao 3o ciclos, 9 foram excluídos por serem professores de Educação Física e 12 encontravam-se licenciados, totalizando 792 professores elegíveis para o estudo. Destes, 751 $(94,8 \%)$ participaram da pesquisa. Os demais $(5,2 \%)$ recusaram-se a participar e os questionários não foram considerados válidos.

A média de idade dos participantes foi de 41 anos (DP = 8), 89,1\% eram mulheres, 55,5\% eram casados ou viviam em união consensual, $66,4 \%$ possuíam filhos, $92,9 \%$ tinham feito pelo menos um curso superior e $45,7 \%$ possuíam uma renda pessoal mensal inferior a $\mathrm{R} \$ 1.600,00$ (Tabela 1).

Sessenta e sete por cento da população estudada consumiam bebidas alcoólicas, 9,8\% eram fumantes, $49,1 \%$ relataram não praticar nenhuma atividade física regular e 9,7\% dos professores relataram usar medicamentos para alterações do sono (Tabela 1).

Com relação ao trabalho, 46,7\% lecionavam em mais de uma escola, $12,1 \%$ exerciam outra função na mesma escola, 10,4\% possuíam uma outra atividade remunerada além do trabalho docente e $45,1 \%$ utilizavam o coletivo urbano como meio de transporte no percurso casa-escola-casa (Tabela 2).

Mais de 50\% tinham tempo de docência superior a 15 anos (média $=16,6$; DP $=8$ anos) e $53,6 \%$ apresentavam carga horária semanal de trabalho docente superior a 22 horas e meia. Quase dois terços (65,6\%) dos professores lecionavam em mais de um turno e $36,8 \%$ em mais de um ciclo (Tabela 2).

Com relação à violência na escola, 15,3\% dos professores relataram episódios de agressão 
Prevalência de transtornos mentais e o perfil da população estudada segundo as características sócio-demográficas, comportamento relacionado à saúde e relato de morbidade crônica.

\begin{tabular}{|c|c|c|c|c|c|c|}
\hline \multirow[t]{2}{*}{ Variável } & \multicolumn{4}{|c|}{ Transtornos mentais } & \multirow[t]{2}{*}{ OR } & \multirow[t]{2}{*}{ IC95\% } \\
\hline & Sim & $\%$ & Não & $\%$ & & \\
\hline \multicolumn{7}{|l|}{ Sexo } \\
\hline Feminino & 339 & 91,1 & 317 & 87,1 & & \\
\hline Masculino & 33 & 8,9 & 47 & 12,9 & 0,66 & $0,41-1,05$ \\
\hline \multicolumn{7}{|l|}{ Faixa etária (anos) } \\
\hline $19-29$ & 27 & 7,3 & 32 & 8,9 & & \\
\hline $30-39$ & 139 & 37,8 & 119 & 33,3 & 1,32 & $0,79-2,44$ \\
\hline $40-49$ & 148 & 40,2 & 134 & 37,4 & 1,31 & $0,75-2,30$ \\
\hline $50 \mathrm{ou}+$ & 54 & 14,7 & 73 & 20,4 & 0,88 & $0,47-1,63$ \\
\hline \multicolumn{7}{|l|}{ Estado civil } \\
\hline Casado ou união consensual & 199 & 53,4 & 211 & 57,7 & & \\
\hline Não casado & 174 & 46,6 & 155 & 42,3 & 1,19 & $0,89-1,59$ \\
\hline \multicolumn{7}{|l|}{ Número de filhos } \\
\hline Nenhum & 121 & 34,3 & 115 & 32,9 & & \\
\hline $1-2$ & 179 & 50,7 & 178 & 50,9 & 0,96 & $0,69-1,33$ \\
\hline 3 ou + & 53 & 15,0 & 57 & 16,2 & 0,88 & $0,56-1,39$ \\
\hline \multicolumn{7}{|l|}{ Escolaridade } \\
\hline Médio & 26 & 7,0 & 26 & 7,1 & & \\
\hline Superior & 190 & 51,5 & 158 & 43,3 & 1,20 & $0,67-2,16$ \\
\hline Superior com especialização & 153 & 41,5 & 181 & 49,6 & 0,85 & $0,47-1,52$ \\
\hline \multicolumn{7}{|l|}{ Renda pessoal mensal (R\$) } \\
\hline$\leq 1.600,00$ & 162 & 43,5 & 174 & 47,8 & & \\
\hline $1.600,00-2.400,00$ & 162 & 43,5 & 132 & 36,3 & 1,32 & $0,96-1,80$ \\
\hline$>2.400,00$ & 48 & 13,0 & 58 & 5,9 & 0,89 & $0,57-1,38$ \\
\hline \multicolumn{7}{|l|}{ Renda total mensal (R\$) } \\
\hline$\leq 1.600,00$ & 70 & 19,0 & 67 & 18,6 & & \\
\hline $1.600,00-2.400,00$ & 133 & 36,0 & 115 & 31,9 & 1,11 & $0,73-1,68$ \\
\hline$>2.400,00$ & 166 & 45,0 & 179 & 49,5 & 0,89 & $0,60-1,32$ \\
\hline \multicolumn{7}{|l|}{ Tabagismo } \\
\hline Não-fumante & 269 & 74,5 & 269 & 74,7 & & \\
\hline Ex-fumante & 52 & 14,4 & 60 & 16,7 & 0,87 & $0,58-1,30$ \\
\hline Fumante & 40 & 11,1 & 31 & 8,6 & 1,29 & $0,78-2,12$ \\
\hline \multicolumn{7}{|l|}{ Atividade física (vezes/semana) } \\
\hline Nenhuma & 190 & 52,3 & 167 & 45,9 & & \\
\hline$\geq 2$ & 103 & 28,4 & 114 & 31,3 & 1,07 & $0,70-1,62$ \\
\hline 3 ou + & 70 & 19,3 & 83 & 22,8 & 1,35 & $0,92-1,97$ \\
\hline \multicolumn{7}{|l|}{ Consumo de bebidas alcoólicas } \\
\hline Não & 115 & 31,0 & 127 & 34,8 & & \\
\hline Sim & 256 & 69,0 & 238 & 65,2 & 1,19 & $0,87-1,62$ \\
\hline \multicolumn{7}{|l|}{ Uso de medicamentos para } \\
\hline \multicolumn{7}{|l|}{ hipertensão arterial } \\
\hline Não & 336 & 90,1 & 317 & 86,1 & & \\
\hline Sim & 37 & 9,9 & 51 & 13,9 & 0,69 & $0,44-1,07$ \\
\hline \multicolumn{7}{|c|}{ Uso de medicamentos para diabetes } \\
\hline Não & 368 & 98,7 & 362 & 98,4 & & \\
\hline Sim & 5 & 1,3 & 6 & 1,6 & 0,82 & $0,30-2,24$ \\
\hline \multicolumn{7}{|c|}{ Uso de medicamentos para distúrbios } \\
\hline \multicolumn{7}{|l|}{ do sono } \\
\hline Não & 315 & 84,5 & 354 & 96,2 & & \\
\hline Sim & 58 & 15,5 & 14 & 3,8 & 4,66 & $2,55-8,51$ \\
\hline Reumatismo & & & & & & \\
\hline Não & 367 & 98,4 & 366 & 99,5 & & \\
\hline Sim & 6 & 1,6 & 2 & 0,5 & 2,99 & $0,60-14,91$ \\
\hline
\end{tabular}


Prevalência de transtornos mentais e características da população estudada segundo a sua inserção no trabalho e carga de trabalho.

\begin{tabular}{|c|c|c|c|c|c|c|}
\hline \multirow[t]{2}{*}{ Variável } & \multicolumn{4}{|c|}{ Transtornos mentais } & \multirow[t]{2}{*}{ OR } & \multirow[t]{2}{*}{ IC95\% } \\
\hline & Sim & $\%$ & Não & $\%$ & & \\
\hline \multicolumn{7}{|c|}{ Trabalha em outra escola } \\
\hline Não & 195 & 53,3 & 194 & 53,3 & & \\
\hline Sim & 171 & 46,7 & 170 & 46,7 & 1,00 & $0,75-1,34$ \\
\hline \multicolumn{7}{|c|}{ Exerce outra função na escola } \\
\hline Não & 322 & 87,5 & 324 & 88,3 & & \\
\hline Sim & 46 & 12,5 & 43 & 11,7 & 1,08 & $0,69-1,68$ \\
\hline \multicolumn{7}{|c|}{ Possui outra função remunerada } \\
\hline Não & 327 & 89,1 & 328 & 90,1 & & \\
\hline Sim & 40 & 10,9 & 36 & 9,9 & 1,12 & $0,69-1,79$ \\
\hline \multicolumn{7}{|c|}{ Meio de transporte utilizado } \\
\hline \multicolumn{7}{|c|}{ para o trabalho } \\
\hline Coletivo & 157 & 44,1 & 163 & 46,0 & 1,08 & $0,80-1,48$ \\
\hline Carro & 199 & 55,9 & 191 & 54,0 & 1,11 & $0,52-2,38$ \\
\hline \multicolumn{7}{|c|}{ Tempo de docência (anos) } \\
\hline $0-4$ & 31 & 8,7 & 33 & 9,4 & & \\
\hline $5-9$ & 48 & 13,4 & 40 & 11,4 & 1,28 & $0,67-2,44$ \\
\hline $10-14$ & 76 & 21,3 & 69 & 19,7 & 1,17 & $0,65-2,11$ \\
\hline $15-19$ & 82 & 23,0 & 75 & 21,4 & 1,16 & $0,66-2,08$ \\
\hline$\geq 20$ & 120 & 33,6 & 134 & 38,1 & 0,95 & $0,55-1,65$ \\
\hline \multicolumn{7}{|c|}{ Tempo de docência na escola (anos) } \\
\hline $0-4$ & 170 & 49,6 & 165 & 49,0 & & \\
\hline $5-9$ & 55 & 16,0 & 63 & 18,7 & 1,09 & $0,72-1,65$ \\
\hline $10-14$ & 64 & 18,7 & 57 & 16,9 & 0,85 & $0,56-1,29$ \\
\hline 15 ou + & 54 & 15,7 & 52 & 15,4 & 1,01 & $0,65-1,56$ \\
\hline \multicolumn{7}{|c|}{ Carga horária semanal (horas semanais) } \\
\hline $0,0-22,5$ & 61 & 17,6 & 74 & 21,7 & & \\
\hline $22,5-45,0$ & 179 & 51,6 & 174 & 51,1 & 1,25 & $0,84-1,86$ \\
\hline$>45,0$ & 107 & 30,8 & 93 & 27,2 & 1,40 & $0,90-2,16$ \\
\hline \multicolumn{7}{|c|}{ Número de ciclos na escola } \\
\hline Apenas um & 221 & 61,9 & 227 & 63,8 & & \\
\hline Mais de um & 136 & 38,1 & 129 & 36,2 & 1,08 & $0,80-1,47$ \\
\hline \multicolumn{7}{|c|}{ Número de turnos na escola } \\
\hline Apenas um & 118 & 32,2 & 131 & 36,2 & & \\
\hline Mais de um & 248 & 67,8 & 231 & 63,8 & 1,19 & $0,88-1,62$ \\
\hline
\end{tabular}

$\mathrm{N}=751$.

praticados por funcionários ou professores, $74 \%$ citaram episódios envolvendo alunos; $57,1 \%$ episódios envolvendo pais de alunos e 54,9\% agressão por pessoas externas à escola (Tabela 3 ).

Quando pesquisados sobre sua percepção acerca do exercício do trabalho docente, 18,8\% relataram ter pouca margem de autonomia, $10,5 \%$ informaram que a margem de criatividade é pequena, $36,4 \%$ relataram ter pouco tempo para o preparo das aulas e $43,5 \%$ informa- ram ter pouco tempo para correção de trabalhos (Tabela 4). O uso de vídeo ou TV nas aulas foi informado por $91 \%$ dos professores. Oitenta e nove por cento consideraram insuficiente o número de computadores para os alunos (Tabela 4).

O ruído elevado na sala de aula foi informado por $48,4 \%$ dos respondentes. Cerca de $45 \%$ queixaram-se de ruído na escola gerado fora da sala de aula e $17 \%$ classificaram como elevado 


\begin{tabular}{|c|c|c|c|c|c|c|}
\hline \multirow[t]{2}{*}{ Variável } & \multicolumn{4}{|c|}{ Transtornos mentais } & \multirow[t]{2}{*}{ OR } & \multirow[t]{2}{*}{ IC95\% } \\
\hline & Sim & $\%$ & Não & $\%$ & & \\
\hline \multicolumn{7}{|l|}{ Agressão por alunos } \\
\hline Nunca & 83 & 22,7 & 106 & 29,4 & & \\
\hline Uma vez & 232 & 63,4 & 227 & 62,9 & 1,31 & $0,93-1,84$ \\
\hline Mais de uma vez & 51 & 13,9 & 28 & 7,7 & 2,33 & $1,35-4,00$ \\
\hline \multicolumn{7}{|c|}{ Agressão por pais de alunos } \\
\hline Nunca & 133 & 36,2 & 177 & 49,7 & & \\
\hline Uma vez & 212 & 57,8 & 168 & 47,2 & 1,68 & $1,24-2,27$ \\
\hline Mais de uma vez & 22 & 6,0 & 11 & 3,1 & 2,66 & $1,25-5,68$ \\
\hline \multicolumn{7}{|c|}{ Agressão por funcionários ou professores } \\
\hline Não & 303 & 82,3 & 315 & 87,0 & & \\
\hline Sim & 65 & 17,7 & 47 & 13,0 & 1,45 & $0,97-2,17$ \\
\hline \multicolumn{7}{|l|}{ Agressão externa } \\
\hline Nunca & 140 & 38,0 & 187 & 52,3 & & \\
\hline Uma vez & 43 & 11,7 & 37 & 10,3 & 1,75 & $1,29-2,36$ \\
\hline Mais de uma vez & 185 & 50,3 & 134 & 37,4 & 2,31 & $1,06-5,00$ \\
\hline
\end{tabular}

o ruído gerado fora da escola. A ventilação na sala de aula foi considerada precária por $23,7 \%$ dos entrevistados, $12 \%$ referiram a iluminação precária e 4,7\% consideraram ruins as condições das paredes das salas de aula (Tabela 4).

A prevalência de transtornos mentais (escore total no GHQ $\geq 4$ ) foi de 50,3\%. O transtorno mental não apresentou associação estatisticamente significante com nenhuma das características sócio-demográficas da população estudada (Tabela 1), nem com nenhuma das variáveis relativas à inserção no trabalho ou carga de trabalho (Tabelas 2).

Com relação ao comportamento e alguns indicadores de saúde, os resultados indicam apenas a associação positiva entre transtornos mentais e o uso de medicamentos para alterações de sono (Tabela 1).

Os resultados mostram uma associação forte e com gradiente de intensidade positivo entre a prevalência de transtornos mentais e todas as variáveis relacionadas à experiência de violência na escola (Tabela 3), à percepção negativa sobre o trabalho (Tabela 4) e às condições do ambiente físico na escola (Tabela 4).

Observaram-se transtornos mentais mais freqüentes nos professores que relataram um ou mais episódios de agressão praticados na escola por alunos, pais de alunos e pessoas externas à escola.
Os resultados mostram que, quanto menor a margem de autonomia maior a prevalência de transtornos mentais nesses professores. $\mathrm{O}$ mesmo ocorre para a possibilidade de ser criativo, para o tempo disponível à preparação de aulas e para o tempo de correção de trabalhos. Neste estudo, a possibilidade de ser criativo foi um fator de proteção, enquanto os demais foram fatores de risco (Tabela 4).

A grande maioria de professores relatou ter disponível para as atividades escolares o uso de TV e vídeo e material atualizado na biblioteca. Uma pequena minoria relatou dispor de computadores em número suficiente para uso dos alunos e acesso à Internet para os mesmos. Apenas a disponibilidade de computadores foi estatisticamente associada como fator de proteção à presença de transtornos mentais (Tabela 4).

Quanto ao ambiente físico e às condições de conforto no trabalho, a análise mostrou um gradiente de associação positiva com maior prevalência de transtorno mental nas piores condições de ruído, ventilação e condições das paredes das salas de aula (Tabela 4 ).

$\mathrm{Na}$ análise de regressão logística múltipla, em que se verificaram os efeitos simultâneos das variáveis, mantiveram-se associadas no bloco sobre violência apenas a agressão por pessoas externas à escola e por pais de alunos; no de percepção sobre o trabalho, a possibilidade 
Prevalência de transtornos mentais e características da população estudada segundo a percepção sobre o trabalho, os recursos disponíveis para o trabalho e ambiente físico escolar.

\begin{tabular}{|c|c|c|c|c|c|c|}
\hline \multirow[t]{2}{*}{ Variável } & \multicolumn{4}{|c|}{ Transtornos mentais } & \multirow[t]{2}{*}{ OR } & \multirow[t]{2}{*}{ IC95\% } \\
\hline & Sim & $\%$ & Não & $\%$ & & \\
\hline \multicolumn{7}{|l|}{ Margem de autonomia } \\
\hline Grande & 75 & 20,3 & 118 & 32,4 & & \\
\hline Razoável & 211 & 57,0 & 196 & 53,8 & 1,69 & $1,19-2,40$ \\
\hline Pequena & 84 & 22,7 & 50 & 13,8 & 2,64 & $1,68-4,16$ \\
\hline \multicolumn{7}{|c|}{ Possibilidade de ser criativo } \\
\hline Grande & 151 & 40,7 & 219 & 59,8 & & \\
\hline Razoável & 165 & 44,5 & 125 & 34,2 & 1,91 & $1,40-2,61$ \\
\hline Pequena & 55 & 14,8 & 22 & 06,0 & 3,63 & $2,12-6,20$ \\
\hline \multicolumn{7}{|c|}{ Tempo para preparação de aulas } \\
\hline Muito & 37 & 10,3 & 61 & 17,8 & & \\
\hline Razoável & 166 & 46,4 & 182 & 53,1 & 1,55 & $1,02-2,33$ \\
\hline Pouco & 155 & 43,3 & 100 & 29,2 & 2,63 & $1,70-4,05$ \\
\hline \multicolumn{7}{|c|}{ Tempo para correção de trabalhos } \\
\hline Muito & 32 & 9,0 & 44 & 12,8 & & \\
\hline Razoável & 141 & 39,5 & 179 & 52,0 & 1,08 & $0,65-1,80$ \\
\hline Pouco & 184 & 51,5 & 121 & 35,2 & 2,09 & $1,26-3,48$ \\
\hline \multicolumn{7}{|l|}{ Uso de TV e vídeo } \\
\hline Não & 35 & 9,4 & 28 & 7,6 & & \\
\hline Sim & 337 & 90,6 & 339 & 92,4 & 1,26 & $0,75-2,11$ \\
\hline \multicolumn{7}{|c|}{ Computadores suficientes para os alunos } \\
\hline Não & 343 & 92,2 & 316 & 86,6 & & \\
\hline $\operatorname{Sim}$ & 29 & 7,8 & 49 & 13,4 & 0,55 & $0,34-0,89$ \\
\hline \multicolumn{7}{|c|}{ Acesso à Internet para os alunos } \\
\hline Não & 307 & 82,5 & 289 & 80,3 & & \\
\hline $\operatorname{Sim}$ & 65 & 17,5 & 71 & 19,7 & 0,86 & $0,59-1,25$ \\
\hline \multicolumn{7}{|c|}{ Biblioteca com material atualizado } \\
\hline Não & 76 & 20,9 & 58 & 15,8 & & \\
\hline $\operatorname{Sim}$ & 287 & 79,1 & 308 & 84,2 & 0,73 & $0,50-1,07$ \\
\hline \multicolumn{7}{|c|}{ Ruído gerado na sala de aula } \\
\hline Desprezível a razoável & 146 & 39,1 & 236 & 64,1 & & \\
\hline Elevado a insuportável & 227 & 60,9 & 132 & 35,9 & 2,78 & $2,06-3,74$ \\
\hline \multicolumn{7}{|c|}{ Ruído gerado na escola fora } \\
\hline \multicolumn{7}{|l|}{ da sala de aula } \\
\hline Desprezível a razoável & 165 & 44,5 & 245 & 67,1 & & \\
\hline Elevado a insuportável & 206 & 55,5 & 120 & 32,9 & 2,55 & $1,89-3,44$ \\
\hline \multicolumn{7}{|c|}{ Ruído gerado fora da escola } \\
\hline Desprezível a razoável & 294 & 79,2 & 317 & 87,1 & & \\
\hline Elevado a insuportável & 77 & 20,8 & 47 & 12,9 & 1,77 & $1,19-2,63$ \\
\hline \multicolumn{7}{|l|}{ Ventilação na sala de aula } \\
\hline Satisfatória & 99 & 26,7 & 119 & 32,4 & & \\
\hline Razoável & 170 & 45,8 & 174 & 47,4 & 1,17 & $0,82-1,70$ \\
\hline Precária & 102 & 27,5 & 74 & 20,2 & 1,70 & $1,11-2,60$ \\
\hline \multicolumn{7}{|l|}{ Iluminação da sala de aula } \\
\hline Satisfatória & 129 & 34,8 & 176 & 48,0 & & \\
\hline Razoável & 197 & 53,1 & 147 & 40,0 & 1,40 & $0,87-2,24$ \\
\hline Precária & 45 & 12,1 & 44 & 12,0 & 1,83 & $1,34-2,50$ \\
\hline \multicolumn{7}{|c|}{ Condições das paredes na sala de aula } \\
\hline Precárias & 24 & 6,5 & 11 & 3,0 & & \\
\hline Razoáveis & 160 & 43,2 & 135 & 36,8 & 0,53 & $0,24-1,20$ \\
\hline Satisfatórias & 186 & 50,3 & 221 & 60,2 & 0,39 & $0,17-0,86$ \\
\hline
\end{tabular}


de ser criativo como fator protetor; e no de ambiente físico, o ruído dentro e fora da escola e a má iluminação (Tabela 5).

\section{Discussão}

O presente estudo mostrou que a prevalência de indivíduos em risco de apresentar transtornos mentais foi bastante elevada nos professores da Regional Nordeste da rede municipal de ensino de Belo Horizonte. Além disso, os resultados mostraram que essas alterações são mais freqüentes nos professores que relataram experiência de violência nas escolas, pior percepção sobre o trabalho, piores condições de trabalho e ambiente físico da escola, e menor recurso computacional para o trabalho escolar. O transtorno mental também foi associado ao uso de medicamentos para dormir. Nenhuma das variáveis relacionadas às características sócio-demográficas, ao tipo de inserção no trabalho e à carga de trabalho apresentaram associação estatística com a prevalência de transtornos mentais.

A prevalência de indivíduos em risco de apresentar transtornos mentais encontrada na amostra foi muito superior àquela encontra em estudos realizados na população geral em outras localidades do país 27 , reforçando o caráter preocupante do problema entre os docentes, sobretudo considerando-se a faixa etária jovem predominante no grupo estudado. A elevada prevalência de transtornos mentais em uma população jovem é um dado preocupante, podendo ser indicativo de um processo de desgaste acelerado que promove uma série de alterações negativas na saúde dos professores 21 .

A freqüência de distúrbios psíquicos encontrada entre os docentes pesquisados em Vitória da Conquista, Bahia, Brasil, foi duas vezes maior que na população geral, sem diferença significativa entre homens e mulheres ${ }^{22}$. Outros estudos sobre a saúde dos professores, nos diversos níveis de ensino, realizados na Bahia, também evidenciaram prevalências elevadas de distúrbios psíquicos em docentes 4,18,21. É possível que a taxa elevada, encontrada no presente trabalho, tenha sido influenciada também pela época de realização da pesquisa (final do último semestre). Ou seja, expressaria uma tendência sazonal de aumento dos transtornos mentais em professores, decorrente do acúmulo progressivo de tarefas e de problemas organizacionais ao longo do ano. Delcor et al. 22 também observaram essa tendência sazonal no estudo dos professores de Vitória da Conquista.

Resultados semelhantes aos nossos também foram encontrados em estudos com docentes em outros países. Tuettemann 28 , na Austrália, encontrou uma prevalência de transtorno mental de 44,6\% em professores de segundo grau. Estudo realizado em Hong-Kong 29, recentemente, também mostrou que a profissão de ensinar é altamente estressante. Cerca de um terço dos professores pesquisados apresentou sinais de estresse e burnout entre os principais problemas de saúde. Outros estudos existentes sobre a saúde dos professores situam os transtornos psíquicos entre as principais queixas de saúde dos docentes e de causas de absenteísmo nas escolas correspondendo, em sua maioria, a quadros depressivos, nervosismo, abuso de bebidas alcoólicas, sintomas físicos sem explicação e cansaço mental, com sérias conseqüências para o desempenho profissional e impacto cada vez maior na função familiar, além de serem responsáveis por elevados custos sociais 22,30,31.

A distribuição da população estudada quanto às características sócio-demográficas, ou seja, o predomínio de mulheres jovens, casadas,

Tabela 5

Fatores que permaneceram estatisticamente associados ao transtorno mental nas análises multivariadas realizadas separadamente para cada conjunto de fatores previamente analisado.

\begin{tabular}{llll}
\hline Conjunto de fatores analisado & Variável & OR & IC95\% \\
\hline Experiência de violência na escola & Agressão externa mais de uma vez & 1,52 & $1,07-2,14$ \\
& Agressão por pais de alunos mais de uma vez & 1,44 & $1,00-2,07$ \\
Percepção sobre o trabalho & Possibilidade de ser criativo & 0,36 & $0,19-0,67$ \\
Ambiente físico da escola & lluminação precária da sala de aula & 1,72 & $1,15-2,56$ \\
& Ruído elevado na escola fora da sala de aula & 1,77 & $1,26-2,50$ \\
& Ruído elevado dentro da sala de aula & 2,24 & $1,62-3,11$ \\
\hline
\end{tabular}


com escolaridade superior e renda mensal de até $\mathrm{R} \$ 2.400,00$, é semelhante aos resultados encontrados em outras pesquisas no país 4,14,19,22. No grupo das professoras da Regional Nordeste encontrou-se uma prevalência de transtornos mentais igual a $51,7 \%$ e entre os professores a taxa foi de $41,3 \%$. Essa diferença não alcançou nível de significância estatística, convergindo com os resultados de Araújo et al. 21 em Salvador e de Tuettemann 28 na Austrália.

A Lei de Diretrizes e Bases da Educação, aprovada em 1996, exige escolaridade superior para todos os níveis de ensino, o que pode explicar a elevada porcentagem de professores da rede municipal de ensino de Belo Horizonte com nível superior. Essa homogeneidade possivelmente explique a ausência de associação do nível de escolaridade com os transtornos pesquisados.

A forte associação entre transtornos mentais e uso de medicamentos para alterações do sono pode ser explicada pelo fato de que a perda de sono é uma expressão freqüente desses distúrbios.

Verificou-se, neste estudo, que pequena margem de autonomia e pequena possibilidade de ser criativo na execução das atividades, da mesma forma que a escassez de tempo para preparo das aulas, apresentaram associação estatística com o risco de apresentar transtornos mentais. Esses resultados são similares aos de outros estudos que encontraram relação de distúrbios psíquicos com alta demanda no trabalho 4,30,31, reforçando a idéia de que alta demanda no trabalho predispõe ao estresse e às suas conseqüências.

Quase metade da população estudada trabalhava simultaneamente em outra(s) escola(s) e metade apresentava carga horária de trabalho semanal entre 22,5 e 45 horas, possivelmente para aumentar a renda mensal. Como o trabalho docente não se restringe à sala de aula, sendo muitas as tarefas realizadas fora da escola, a extensão da jornada de trabalho docente pode gerar uma carga bem superior àquela formalmente indicada nos contratos. Reis 4 sugere que o desgaste físico e psíquico pode ser desencadeado ou agravado por uma jornada de trabalho prolongada. Entretanto, não encontramos associação entre transtornos mentais e variáveis indicadoras de carga de trabalho neste estudo. É possível que as variáveis selecionadas não tenham discriminado adequadamente essa carga, mas também é razoável pensar que, na população estudada, existem outros fatores mais relevantes para a saúde mental dos professores, no momento em que se realizou a pesquisa.
O relato de violência nas escolas foi muito freqüente entre os professores estudados. Todas as formas de agressões praticadas por alunos, pais de alunos, funcionários ou colegas de trabalho, ou pessoas externas à escola, foram fortemente associadas aos transtornos mentais. Esses resultados são coerentes com o estudo de Schonfeld 32 , realizado com professoras na cidade de Nova Iorque, Estados Unidos, que também mostrou uma forte associação de sintomas depressivos e ambientes de trabalho nocivos, perigosos ou carentes de controle.

Os dados corroboram as afirmações da OIT e UNESCO sobre o aumento da violência nas instituições escolares em todo o mundo 11,23. Esteve 11 sugere que, além do efeito direto da agressão, deve-se levar em conta o efeito multiplicador dessas experiências no campo psicológico, sobre os colegas ou amigos do professor agredido, totalmente alheios à cena de ação mas que recebem o impacto através dos meios de comunicação social. Para Abramovay \& Ruas 23 , a escola não é mais representada como um local seguro de integração social, de socialização, não é mais um espaço resguardado; tornou-se cenário de ocorrências violentas.

Neste estudo, os professores que relataram como insuficiente o número de computadores disponíveis para os alunos apresentaram maior prevalência de transtornos mentais. O resultado encontrado é coerente com outras investigações sobre o tema. A falta ou escassez de recursos materiais e condições adversas no ambiente de trabalho incidem diretamente sobre a ação docente, sendo considerados como fatores geradores de tensões negativas em sua prática cotidiana 33,34. A carência de recursos observada torna-se contraditória às diretrizes do sistema educacional que promovem uma renovação metodológica sem, ao mesmo tempo, suprir os professores dos recursos necessários para atendê-las 11 .

A deficiência de ventilação na sala de aula e a presença de ruído, seja ele gerado na sala de aula, seja fora da escola, são condições ambientais adversas à execução das atividades docentes e estiveram associadas ao transtorno mental. Esses fatores tornam o ambiente desconfortável, incômodo e intranqüilo, para professores e alunos, requerendo maior esforço e maior exigência física e mental com repercussões negativas sobre a saúde. É possível também que os professores com transtornos mentais tenham uma percepção exacerbada sobre o ruído e as condições de conforto em geral. Segundo Figueroa et al. 34 , os aspectos físicos do ambiente de trabalho têm um lugar preponderante na origem do mal-estar. Uma longa jor- 
nada de trabalho em ambiente com níveis elevados de ruído pode induzir ao estresse e causar sintomas como dor de cabeça e irritabilidade.

Embora o instrumento construído para esta pesquisa tenha contemplado um bloco de questões que abordam os relacionamentos dos professores com alunos, com pais de alunos, com dirigentes, com os colegas e demais funcionários da escola, não foi possível estudá-las porque um grande número de professores, cerca de metade dos participantes, não respondeu a essas questões. Acredita-se que esse fato foi influenciado pela estratégia de coleta de dados. Em muitas escolas não foi possível aplicar os questionários no horário da reunião pedagógica. A alternativa foi deixá-los na escola, aos cuidados da coordenação ou da direção, retornando, em outro momento, para buscá-los. É possível que os professores tenham temido uma quebra no anonimato dos questionários e, por isso, não tenham respondido a essas questões. Vários autores citam os relacionamentos interpessoais, internos ou externos, como extremamente importantes para a satisfação com o trabalho, existindo fortes evidências de que relações sociais conflituosas podem afetar a saúde, aumentando a carga de trabalho e conseqüente desgaste físico e mental 11,14,15,20.

A participação no presente estudo foi eleva$\mathrm{da}$, o que garante a validade interna dos resultados encontrados. Entretanto, é preciso ressaltar que o estudo apresenta algumas limitações. A primeira diz respeito à exclusão dos trabalhadores afastados por licença médica, o que introduz o viés do trabalhador sadio, que tende a subestimar a real prevalência de transtornos mentais na população de estudo. Como descrito por Gasparini et al. 35, muitos professores estão afastados justamente por problemas de saúde mental. A segunda limitação importante refere-se ao corte transversal do desenho de estudo, que não permite inferir uma relação de natureza causal entre transtornos mentais e os fatores associados aos mesmos.

Concluindo, o presente estudo identificou uma alta prevalência de transtorno mental entre docentes da rede municipal de ensino fundamental de Belo Horizonte. É possível que esses distúrbios estejam associados às variáveis identificadas neste estudo. Mas é possível, tam- bém, que essas variáveis estejam agravando problemas preexistentes ou que elas sejam mais facilmente notadas por pessoas que têm transtornos mentais e, por isso, são mais freqüentemente relatadas por elas. Os resultados apontam para a necessidade de avançar os estudos sobre esses transtornos e sobre o trabalho docente, para se compreender melhor as associações encontradas e subsidiar propostas que contribuam para elevar a satisfação no trabalho e melhorar a saúde mental dos professores.

\section{Recomendações}

A elevada prevalência de transtornos mentais em professores, determinada neste estudo, e sua associação com os fatores investigados apontam para a necessidade de ações que melhorem as condições do trabalho docente, entre as quais podem ser citadas:

- Estímulo a outros estudos que aprofundem a investigação e que possam avançar na compreensão do papel dos fatores identificados no desenvolvimento e evolução dos problemas mentais em professores. Estudos longitudinais em docentes permitiriam compreender melhor como tais condições se inter-relacionam ao longo do tempo, visto que os transtornos mentais são condições recorrentes e que apresentam uma enorme variedade de expressões clínicas. Sugere-se, também, aprofundamento de questões relacionadas à violência e à organização do trabalho, o que pode ser conduzido por estudos qualitativos;

- Promoção de espaços de discussão entre os trabalhadores docentes e gestores com vistas a subsidiar políticas que forneçam condições favoráveis ao exercício da docência, incluindo as questões relacionadas à autonomia, à criatividade e à disponibilidade de recursos materiais;

- Desenvolvimento de um plano de ação voltado para a adequação acústica, térmica e luminosa das escolas municipais, indicando pistas para futuros projetos arquitetônicos;

- Início de um debate para esclarecer e qualificar as experiências de violência vivenciadas pelos professores numa perspectiva interdisciplinar e multiprofissional, articulada a outras ações coletivas e setores sociais relevantes. 


\section{Resumo}

Estudos realizados em todo o mundo evidenciam que os educadores correm o risco de sofrer esgotamento fisico ou mental, em face das dificuldades materiais e psicológicas associadas ao exercício da atividade docente. Objetivou-se estimar a prevalência de transtornos mentais em professores da rede municipal de ensino de Belo Horizonte, Minas Gerais, Brasil, e investigar a associação com as características do trabalho docente. Empregou-se um estudo do tipo corte transversal em professores do ensino fundamental da Regional Nordeste, utilizando-se um questionário auto-aplicado, com seis blocos de questões. O General Health Questionnaire (GHQ) na versão "12" foi o instrumento de rastreamento de transtornos mentais não-psicóticos na população estudada. Participaram do estudo, 751 dos 792 professores do ensino fundamental de vinte e seis escolas municipais da regional citada $(94,8 \%)$. Os transtornos mentais foram significativamente associados à experiência com a violência e piores condições ambientais, ambiente físico e conforto no trabalho, e organizacionais, margem de autonomia, de criatividade e tempo no preparo das aulas. Os resultados apontam para uma situação grave relativamente à saúde da população pesquisada e fornecem elementos consistentes para a proposição de medidas com vistas à melhoria das condições de trabalho docente.

Saúde Mental; Transtornos Mentais; Docentes; Violência; Escolas

\section{Colaboradores}

As três autoras participaram de todas as etapas da pesquisa e da redação do artigo.

\section{Agradecimentos}

Este estudo não teria sido realizado sem a colaboração do Sindicato Único dos Trabalhadores do Ensino, que trouxe a demanda à Universidade Federal de Minas Gerais e viabilizou a entrada dos pesquisadores nas escolas.

\section{Referências}

1. Jacques MGC. Abordagens teórico-metodológicas em saúde/doença mental \& trabalho. Psicol Soc 2003; 15:97-116.

2. Karasek RA. Job demands, job decision latitude, and mental strain: implications for job redesign. Adm Sci Q 1979; 24:285-308.

3. Bongers PM, De Winter CR, Kompier MAJ, Hildebrandt VH. Psychosocial factors at work and musculoskeletal disease. Scand J Work Environ Health 1993; 19:297-312.

4. Reis EJB. Trabalho e saúde mental em professores [Tese de Doutorado]. Salvador: Faculdade de Medicina, Universidade Federal da Bahia; 2004.

5. Martinez MC. A análise ergonômica do trabalho no estudo das relações entre os fatores psicossociais e a satisfação no trabalho. In: Anais ABERGO [CD-ROM]. São Paulo: Associação Brasileira de Ergonomia; 2001.

6. Sautier SL, Hurrell Jr. JJ, Murphy LR, Levi L. Factores psicosociales y de organización. In: Enciclopedia de salud y seguridad en el trabajo. http:// www.mtas.es/insht/EncOIT/tomo2.htm\#p5 (acessado em 20/Fev/2005).

7. Abrahão JI, Pinho DLM. As transformações do trabalho e desafios teórico-metodológicos da Ergonomia. Estud Psicol (Natal) 2002; 7:45-52.

8. Oliveira DA, organizadora. Reformas educacionais na América Latina e os trabalhadores docentes. Belo Horizonte: Editora Autêntica; 2003.

9. Oliveira DA. A reestruturação do trabalho docente: precarização e flexibilização. Educação \& Sociedade 2004; 25:1127-44.

10. Estryn-Behar M, Kaminski M, Peigne E, Bonnet N, Vaichere E, Gozlan C, et al. Stress at work and mental health status among female hospital workers. Br J Ind Med 1990; 47:20-8.

11. Esteve JM. O mal-estar docente: a sala de aula e a saúde dos professores. Bauru: Edusc; 1999.

12. Glina, DMR, Rocha LE, Batista ML, Mendonça MGV. Saúde mental e trabalho: uma reflexão sobre o nexo com o trabalho e o diagnóstico, com base prática. Cad Saúde Pública 2001; 17:607-16.

13. Araújo TM, Silvany Neto AM, Reis EJFB, Kavalkievicz C. Condições de trabalho e saúde dos professores da rede particular de ensino. Salvador: Sindicato dos Professores no Estado da Bahia/Universidade Federal da Bahia/Confederação Nacional dos Trabalhadores em Estabelecimentos de Ensino; 1998.

14. Codo W, organizador. Educação: carinho e trabalho. Petrópolis: Editora Vozes/Brasília: Confederação Nacional dos Trabalhadores em Educação; 1999.

15. Tolosa DER. Estudo da organização do trabalho, sentimentos, valorização e expectativa profissional dos professores do 1o e 2o graus da Cidade de Jundiaí, SP [Tese de Doutorado]. Campinas: Universidade Estadual de Campinas; 2000.

16. Fonseca CCOP. O adoecer psíquico no trabalho do professor de ensino fundamental e médio da rede pública do Estado de Minas Gerais [Dissertação de Mestrado]. Florianópolis: Universidade Federal de Santa Catarina; 2001. 
17. Noronha MMB. Condições do exercício profissional da professora e dos possíveis efeitos sobre a saúde: estudo de casos das professoras do Ensino Fundamental em uma Escola Pública de Montes Claros, Minas Gerais [Dissertação de Mestrado]. Belo Horizonte: Faculdade de Medicina, Universidade Federal de Minas Gerais; 2001.

18. Oliveira MG. Condições de trabalho, gênero e saúde: sofrimento e estresse. Um estudo de caso com os profissionais docentes do ensino superior privado de Belo Horizonte [Dissertação de Mestrado]. Belo Horizonte: Pontifícia Universidade Católica de Minas Gerais; 2001.

19. Silvany Neto AM, Araújo TM, Kawalkievicz C, Dutra FRD, Azi GR, Alves RL, et al. Condições de trabalho e saúde dos professores da rede particular de ensino, Salvador, Bahia. Rev Baiana Saúde Pública 2000; 24:42-56.

20. Gomes L. Trabalho multifacetado de professores/ as: a saúde entre limites [Dissertação de Mestrado]. Rio de Janeiro: Escola Nacional de Saúde Pública, Fundação Osvaldo Cruz; 2002.

21. Araújo TM, Reis EJFB, Kavalkievicz C, Silvany Neto AM, Paranho I, Carvalho FMP, et al. Saúde e trabalho docente: dando visibilidade aos processos de desgaste e adoecimento docente a partir da construção de uma rede de produção coletiva. Educação em Revista 2003; (37):183-212.

22. Delcor NS, Araújo TM, Reis EJFB, Porto LA, Carvalho FM, Silva MO, et al. Condições de trabalho e saúde dos professores da rede particular de ensino de Vitória da Conquista, Bahia, Brasil. Cad Saúde Pública 2004; 20:187-203.

23. Abramovay M, Ruas MG, organizadores. Violências nas escolas. Brasília: Organização das Nações Unidas para a Educação, a Ciência e a Cultura; 2004.

24. Yuan-Pang W. Manifestações psicopatológicas não-psicóticas em uma amostra da comunidade chinesa da Cidade de São Paulo [Tese de Doutorado]. São Paulo: Faculdade de Medicina, Universidade de São Paulo; 2002.
25. Goldberg DP, Gater R, Sartorius N, Ustun TB, Piccinelli $\mathrm{M}$, Gureje $\mathrm{O}$, et al. The validity of two versions of the GHQ in the WHO study of mental illness in general health care. Psychol Med 1997; 27:191-7.

26. McDowell I, Newell C. Measuring health: a guide to rating scales and questionnaires. Oxford: Oxford University Press; 1996.

27. Vorcaro CM, Lima-Costa MF, Barreto SM, Uchoa E. Unexpected high prevalence of 1-month depression in a small Brazilian community: the Bambuí Study. Acta Psychiatr Scand 2001; 104:257-63.

28. Tuettmann E. Teaching: stress and satisfaction. Issues in Educational Research 1991; 1:31-42.

29. Chan DW. Hardiness and its role in the stressburnout relationship among prospective Chinese teachers in Hong Kong. Teaching and Teacher Education 2003; 19:381-95.

30. Tennant C. Work related stress and depressive disorders. J Psychosom Res 2001; 51:697-704.

31. Siqueira MJT, Ferreira ES. Saúde das professoras das séries iniciais: o que o gênero tem a ver com isso? Psicol Ciênc Prof 2003; 23:76-83.

32. Schonfeld IS. A longitudinal study of occupational stressors and depressive symptoms in first-year female teachers. Teaching and Teacher Education 1992; 8:151-8.

33. Gonçalves GBB. Uso profissional da voz em sala de aula e organização do trabalho docente [Dissertação de Mestrado]. Belo Horizonte: Faculdade de Educação, Universidade Federal de Minas Gerais; 2003.

34. Figueroa NL, Shufer M, Muiños R, Marro C, Coria EA. Um instrumento para avaliação de estressores psicossociais no contexto de emprego. Psicol Reflex Crit 2001; 14:653-9.

35. Gasparini SM, Barreto SM, Assunção AA. O professor, as condições de trabalho e os efeitos sobre sua saúde. Educação e Pesquisa 2005; 31:189-99.

Recebido em 04/Ago/2005

Versão final reapresentada em 21/Fev/2006

Aprovado em 20/Abr/2006 